\title{
Business Rules Management and Decision Mining - Filling in the Gaps
}

\author{
Sam Leewis \\ HU University of Applied Sciences \\ Utrecht \\ $\underline{\text { sam.leewis@hu.nl }}$
}

\author{
Koen Smit \\ HU University of Applied Sciences \\ Utrecht \\ koen.smit@hu.nl
}

\author{
Matthijs Berkhout \\ HU University of Applied Sciences \\ Utrecht \\ matthijs.berkhout@hu.nl
}

\begin{abstract}
Proper decision-making is one of the most important capabilities of an organization. Adequately managing these decisions is therefore of high importance. Business Rules Management (BRM) is an approach that helps in managing decisions and underlying business logic. However, questions still arise if the decisions are properly improved based on decision data. Decision Mining (DM) could complement BRM capabilities in order to improve towards effective and efficient decision-making. In this study, we propose the integration of BRM and DM through a simulation using a government and a healthcare case. During this simulation, three entry points are presented that describe how decision-related data should be utilized between BRM capabilities and DM phases to be able to integrate them. The presented results provide a basis from which more technical research on the three DM phases can be further explored.
\end{abstract}

\section{Introduction}

Proper decision-making is one of the most important capabilities of an organization [5]. In the previous decades, decision making was only executed by human actors. However, given the technical developments in computer hard- and software, the possibilities to automate decision-making increases. Examples of techniques applied during (semi-) automated decision making are business rules systems, expert systems, and neural networks [25]. To achieve proper decision-making, organizations must design and execute and manage their business decisions, decision logic, and decision-making processes.

One approach to do so is referred to as Business Rules Management (BRM). BRM can be defined as a systematic and controlled approach that supports the elicitation, design, specification, verification, validation, deployment, execution, evaluation, and governance of business decisions and business logic [6, 14, 18, 25]. Business logic can be defined as: " $A$ collection of business rules, business decision tables, or executable analytic models to make individual business decisions" [16]. Separating business logic from other information system concerns (e.g., data, user interface, and process concerns) is in line with earlier conclusions provided by $[6,10,14,30]$.

BRM is often utilized in regulated industries such as banking, insurance, government and medical. As more organizations digitize and (semi-) automate their decision-making by using BRM, the overall maturity of BRM practices rises. This means that a lot of organizations are able to execute and manage their decisions using digitization. However, the question arises whether these organizations are properly managing their BRM capabilities and if the decisionmaking is effectively and efficiently improved upon.

One development that follows the rising level of maturity in the BRM-related research domain is Decision Mining (DM) [12]. Decision mining is "the method of extracting and analyzing decision logs with the aim to extract information from such decision logs for the creation of business rules, to check compliance to business rules and regulations, and to present performance information" [12]. DM is similar to Process Mining (PM) in that it allows for the extraction of information from logs to improve an IS artefact, which in this case are digitized and (semi-) automated decisions an organization executes and manages. This is done by managing three phases, being 1) Discovery, 2) Conformance Checking, and 3) Improvement.

The concept of mining decisions is not new $[2,12$, $17,21]$, however, to the knowledge of the authors, little research has been conducted on how DM transacts with BRM capabilities. We see a similar pattern in practice, where Business Rule Management Systems (BRMS) do not yet effectively enable integration with DM algorithms to ensure improvement. A similar challenge is also observed in the Business Process Management (BPM) field in which existing (open source) BPM software offers limited options to integrate PM algorithms in conjunction with BPM capabilities [4].

The gap in current literature and practice is interesting given the fact that both BRM and DM process business decisions and underlying business 
logic with the goal to improve decision-making at organizations $[12,15,18,23]$, and should therefore be tackled. In this paper, we aim to do this by conceptually integrating the BRM capabilities and DM phases as well as by demonstrating probable entry points for DM output to be used as BRM input for managing business decisions. To do so, we aim to achieve the following research goal: To demonstrate how the phases of DM could be integrated with the BRM capabilities.

The remainder of this paper is structured as follows. The next section presents both the BRM and DM viewpoints and their underlying capabilities (BRM) and phases (DM) in more detail in the form of the background and related work. This is followed by a proposed integration between both domains. Then, the research method is presented. Based on the proposed integration and the research method, the next section utilizes two running examples to demonstrate the integration of BRM and DM in a practical setting. Next, the study and its results are discussed, future research directions are presented and the paper is concluded in the following sections.

\section{Background and Related Work}

Both BRM and DM are addressed in this paper from the viewpoint of the BRM capabilities, to be able to effectively integrate them from a conceptual level. In our study, we adhere to the definition of a capability as: "An ability that an organization, person, or system, possesses" [29]. How a capability is realized by an organization depends on the situation in that specific organization, i.e., what technology or tooling is available, the maturity of the available technology, the available knowledge, and the available resources. A capability can be delivered by a single software application as well as a specific mix of people, processes, and technology. To ground the proposed integration between BRM capabilities and DM phases in the next section, we shortly summarize them here.

\section{Business Rules Management}

The definition of BRM provided in the previous section presents a total of nine capabilities that should be taken into account by organizations to ensure a systematic and controlled approach managing their business decisions and business logic, see Figure 1.

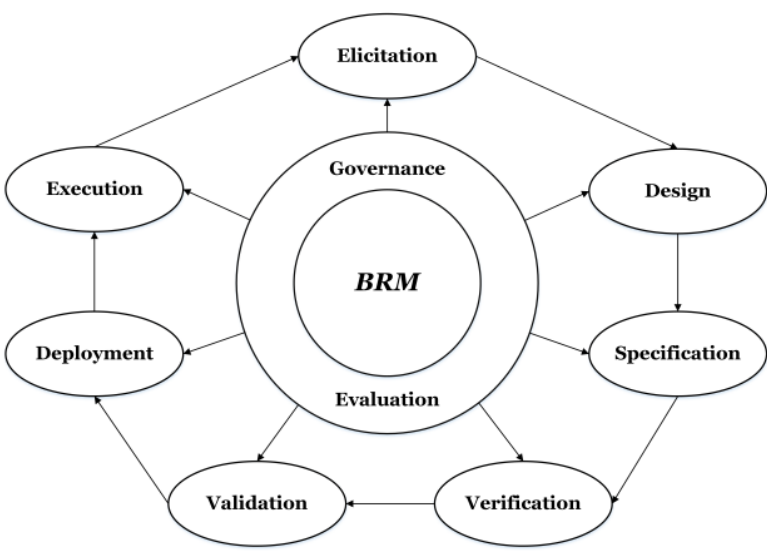

Figure 1. BRM capabilities [25].

The goal of the first capability, Elicitation, is twofold; either to create a new decision or to modify an existing decision [25]. In both situations of creating a new decision service and modifying an existing decision service the scope needs to be clear, thus should be defined as a starting point. The elicitation is triggered by incoming requirements from a client that demands the development of a certain product or service. Therefore, an organization needs to determine the relevant knowledge that needs to be captured from various legal sources to realize the value proposition, i.e., the product or service. Different types of legal sources from which decisions need to be derived are: laws, regulations, policies, internal documentation and human experts [25]. Another situation in which the output for this capability is different is when an impact assessment must be conducted in order to determine the changes in the sources that affect the business decisions.

The goal of the second capability is to create a business rules architecture based on the relevant knowledge derived in the elicitation capability [25]. To be able to do so, an organization needs to structure the knowledge into business decisions and underlying business logic, which are multiple artifacts described in the work of $[24,25]$. The output of this capability is a business decision architecture such as can be modelled using the Decision Model and Notation (DMN) standard in layer one (the decision-layer) [25].

The goal of the specification capability is to define the business logic, e.g., business rules or decision tables, the facttypes, and fact values for each business decision. The output of this capability is a complete set of a business decision with underlying business logic to be verified and validated.

The goal of the verification capability is to determine if the artifacts adhere to predefined criteria and are logically consistent [6]. Verification errors not properly addressed could result in the improper execution of a business decision in the execution 
capability, thus posing a possible risk to the organization that employs the business logic $[27,28]$.

The goal of the validation capability is to determine whether the verified business decisions deliver their intended behavior [31]. In contrast to the verification capability that can be conducted by a BRMS, the validation capability is often conducted by subject matter experts manually. Validation errors not properly identified or addressed could lead to non-compliance, which poses organizations with various risks, e.g., legal fines, civil fines, re-engineering costs, public harms, consumer churn, and loss of public trust [7].

The goal of the deployment capability is to transform the verified and validated business decisions to systems with implementation-dependent languages, e.g., Java, Blaze, or even natural language for human actors to apply in processes. The output of this capability is ready to be executed by the organization.

The goal of the execution capability is to execute the business decisions, which are embedded in the products and services of an organization, e.g., a mortgage calculator or a web app to register for governmental benefits.

The governance capability is a capability that is utilized to parallel the aforementioned capabilities. The goal of this capability is to ensure proper traceability, validity and version management across processes and BRM artefacts used in the aforementioned capabilities $[25,26]$.

The last capability, evaluation, has the goal of monitoring the execution of the business decisions, as well as to monitor the performance of the other capabilities [24] and is also utilized to parallel the other capabilities, similar to the governance capability.

\section{Decision Mining}

Decision mining consist of three phases, 1) the discovery, 2) conformance checking, and 3) improvement of decisions [12], as shown in Figure 2. These phases focus on the estimation of data quality and interpretation of their semantics, interpretation of relevant data, the actual meaning of the data, and the unit of measurement [22]. Furthermore, classifying business decisions allows for the discovery of correspondence between different roles of a decision maker in the development of decision architectures [22]. Decision mining shares a common ground with the field of PM. The activities are similar, however, PM focuses on sequence patterns while DM focuses on derivation patterns [1, 12]. The techniques used for DM and PM are inherently created for mining sequence patterns (PM) and derivation patterns (DM). Multiple studies were conducted from a process mining perspective in order to try to mine decisions from event logs $[3,13,17$, 20, 21].

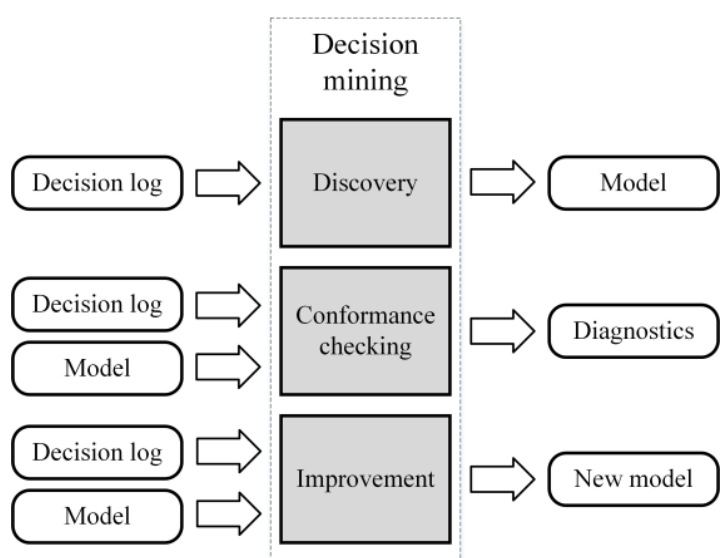

Figure 2. Decision mining [12]

To be able to utilize DM, one key criterion needs to be adhered to, which is the availability of a decision log. A decision log is required to discover decisions from, in order to perform conformance checking, comparing the decision $\log$ to a model, and to utilize the decision log in comparison to a model in order to provide possible improvements to the model [19]. A decision log consists of structured data that is characterized by implicit dependencies between variables. More specific, a combination of conditions resulting in a conclusion, where a conclusion, in turn, could be a condition in another business decision. This is different to, e.g., an event log, which consist of sequence related data [1].

For every DM phase, the decision log serves as an input. The discovery phase utilizes a decision log in order to discover decisions. The output of the Discovery phase is a business decision architecture, e.g., a DRD, as well as decision tables, and business rules. This could be generated in different modelling languages. For this study Decision Model and Notation (DMN) [16] is specified as the output modelling language. The decision model and notation (DMN) is an industry standard that is used for the modeling of business decisions [16].

The conformance checking phase utilizes a decision $\log$ and compares this to a business decision architecture, in order to provide diagnostics if any business decisions are executed (decision log) versus whether this was the intention (the model).

The improvement phase focuses on providing any possible improvement to the model. A new model is provided based on the combination of the decision log and the decision model. 


\section{Proposed integration}

To demonstrate how the BRM capability framework can be completed in an effective manner using DM, we propose several entry points in relation to the BRM capabilities [24]. These entry points are matched with the DM phases and the possibility of supporting or possibly replacing certain activities in the BRM capabilities, see Figure 3. To ground the proposed integration, we specify the BRM capabilities in more detail in this section.

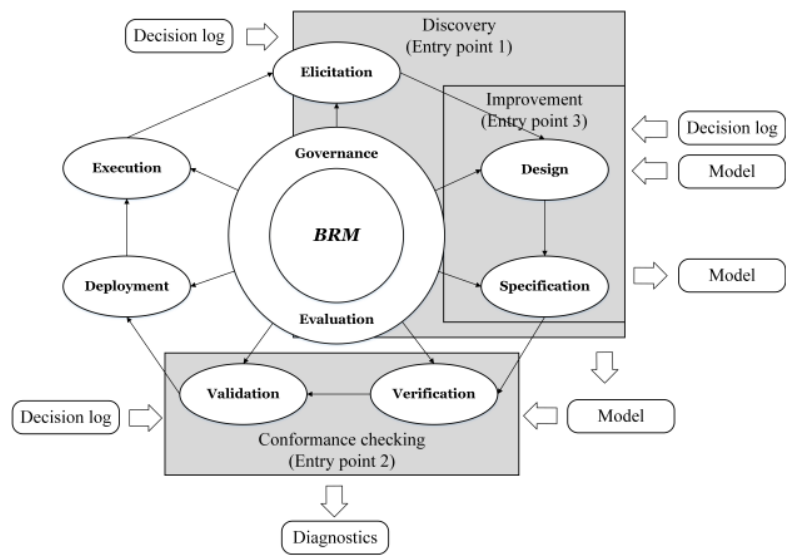

Figure 3. Decision mining entry points in BRM capabilities

Additionally, to fill in the gaps for a single situation in which BRM is applied, multiple information systems could be connected with DM phases, as shown in Figure 4. Different BRMSs could be connected to each other by utilizing a decision log from one BRMS as input to the Discovery phase of DM in order to utilize the model as input for another BRMS.
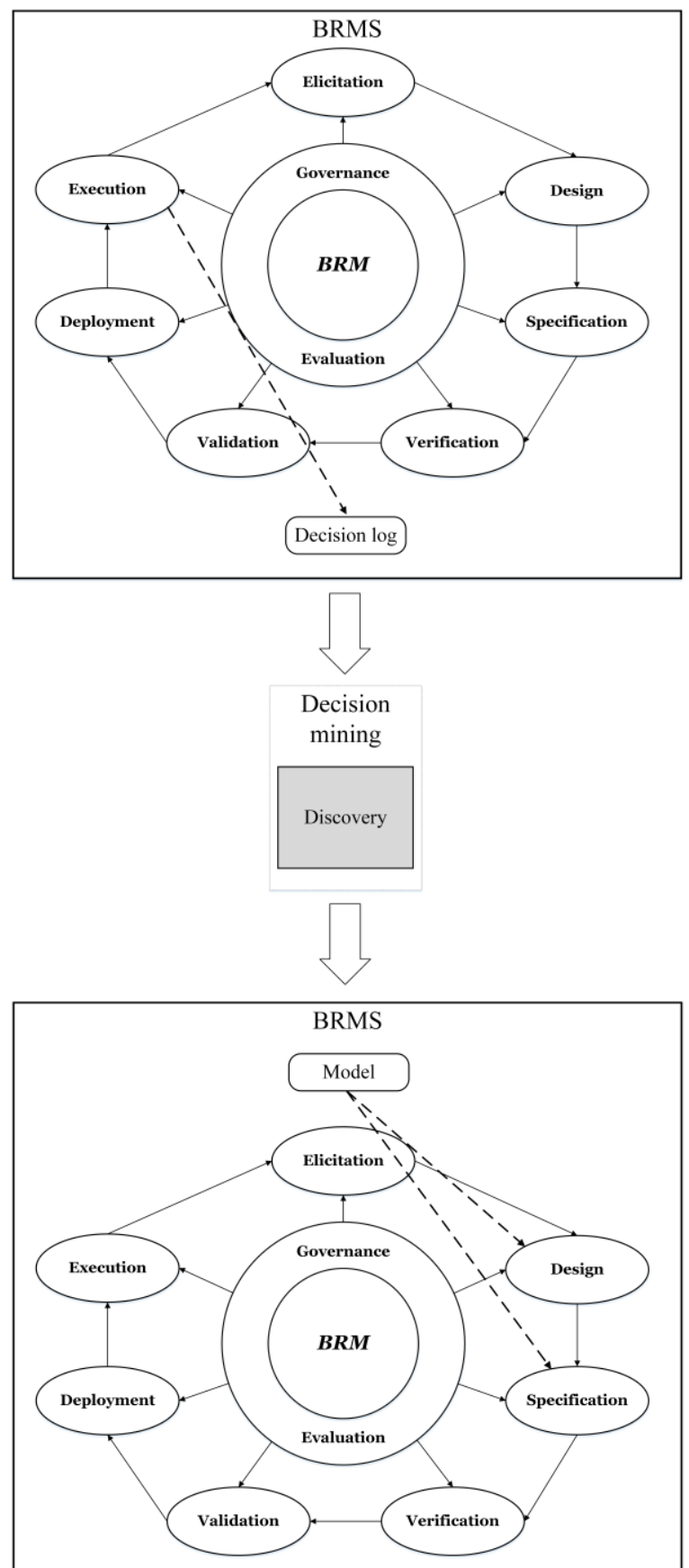

Figure 4. BRMSs connected through Decision mining

Entry point 1: The DM discovery phase has a functional overlap with the BRM elicitation, design and specification capabilities, as shown in Figure 3. Discovery supports the BRM capabilities in the design and specification of a business decision architecture by creating a new model using decision logs.

Entry point 2: The DM conformance checking phase had a functional overlap with the verification and validation BRM capabilities, as shown in Figure 3. 
Conformance checking supports the BRM capabilities in checking an existing model on conformance with a discovered model from a decision log.

Entry point 3: The DM improvement phase has a functional overlap with the design and specification BRM capabilities, as shown in Figure 3. Improvement supports the same capabilities as with entry point 1 , the difference lies with that entry point 1 results into a newly discovered model, and entry point 3 results into a improved model.

In the following section, we first describe, from a methodological point of view, how the entry points are demonstrated, which is followed by two real-life cases to demonstrate how the DM phases and BRM capabilities could enhance each other.

\section{Research Method}

The goal of this study is to identify how the DM phases and BRM capabilities can strengthen each other. To achieve this, we demonstrate, through the proposition of possible entry points, how the DM phases could strengthen the BRM capabilities. Therefore, a simulation method is utilized in this study. Important to mention is that simulation, in this study, is not utilized in a computational manner, but rather as a conceptual approach so that the conceptual fit between DM phases and BRM capabilities can be demonstrated.

Simulation focuses on theory development rather than theory testing. Simulation research provides superior insight into complex theoretical relationships among constructs, especially when challenging empirical data exists [9], which is the case in the context of DM used in combination with BRM [12]. To do so, we apply a simulation in which we introduce two cases constructed from real-life data. The cases feature a decision log and feature conditions and conclusions, which are not altered in any way. However, the data and specific instances shown in the examples are generated to ensure the anonymity of personal data in the original logs as well as to mitigate possible fraud with business decisions and their underlying business logic.

The fit between DM phases and BRM capabilities is demonstrated via the entry points described in the previous section. It is further structured using the three DM phases (discovery, conformance checking, and improvement).

\section{Demonstration}

To demonstrate the integration of BRM and DM, two example cases are presented. The first case comprises a healthcare context whereas the second case comprises a governmental context.
The cases are not the same and will thereby demonstrate the different entry points in the proposed integration. Using the two cases the different entry points in the BRM capabilities are demonstrated, supported by the DM phases.

The healthcare case (hereafter referred to as: "Cardiovascular Risk Case") is based on the work of [8] and is a simulated decision log output of an healthcare information system, e.g., an Electronic Patient Dossier (EPD). The decision log contains data about calculating the risk of cardiovascular disease chances, separated by region. It consists of two decisions where the first decision 'Determine Cardiovascular Risk' has six conditions: Diabetes Mellitus Status (dm), Gender (gdr), Smoking (smk), age, Systolic Blood Pressure (sbp), and Total Cholesterol (chl). The second decision 'Determine Region Specific Cardiovascular Risk' consists of two conditions: the risk score which is the output from 'Determine Cardiovascular Risk' and the Region. The Region consists of the WHO epidemiological regions. This decision has an output of the calculated risk of a patient for cardiovascular disease within a specific region.

\begin{tabular}{|c|c|c|c|c|c|c|c|}
\hline 으 & $\underline{\varepsilon}$ & 产 & है & 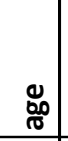 & $\frac{\circ}{n}$ & 든 & 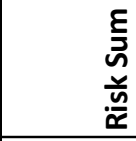 \\
\hline A01 & 1 & 0 & 1 & 40 & 120 & 4 & 19,63756 \\
\hline A02 & 1 & 0 & 1 & 40 & 120 & 5 & 19,8883 \\
\hline A03 & 1 & 0 & 1 & 40 & 120 & 6 & 20,09318 \\
\hline A04 & 1 & 0 & 1 & 40 & 120 & 7 & 20,2664 \\
\hline A05 & 1 & 0 & 1 & 40 & 120 & 8 & 20,41645 \\
\hline A06 & 1 & 0 & 1 & 40 & 140 & 4 & 19,79171 \\
\hline 으 & & & $\begin{array}{l}\frac{\varepsilon}{5} \\
\tilde{n} \\
\frac{y}{u} \\
\frac{a}{x}\end{array}$ & & & ִָ & 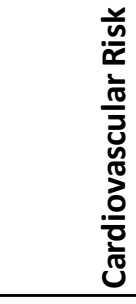 \\
\hline A01 & & 9,63 & 3756 & AFR & R_D & & $10 \%$ \\
\hline A02 & & 19,8 & 8883 & AFR & R D & & $10 \%$ \\
\hline A03 & & 0,09 & 9318 & AM & R_A & & $10 \%$ \\
\hline A04 & & 20,2 & 2664 & AM & R_A & & $10 \%-20 \%$ \\
\hline A05 & & 0,41 & 1645 & AM & IR_A & & $20 \%-30 \%$ \\
\hline A06 & & 9,79 & 9171 & EUR & R_A & & $10 \%$ \\
\hline
\end{tabular}

Figure 5. Cardiovascular Risk Case decision output

The government case is provided by the Dutch Education Executive Agency and is a database output. The database output is a result of the teacher grant 
decision. The teacher grant decision consists of two sub decisions. A decision in the form of a loan for the student's study costs and a related part compensation for study leave to the employer Study leave can only be requested and granted if a loan is also applied for. The government case (hereafter referred to as: "Dutch Teacher Grant case") is an example of a generic dataset with the structure required for a decision log. This dataset is not the outcome of a BRMS. Therefore, to demonstrate the usefulness of DM, such a dataset is selected for this demonstration.

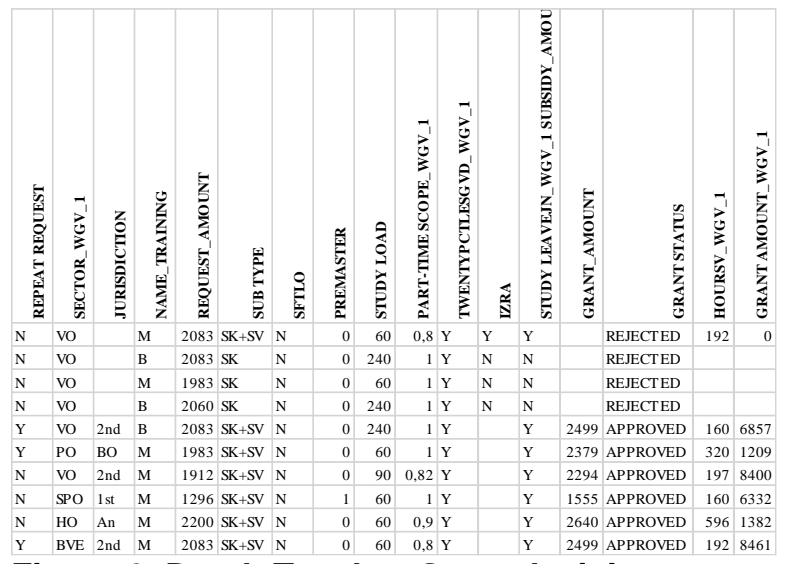

Figure 6. Dutch Teacher Grant decision output

\subsection{Discovery entry point}

Starting with the Discovery entry point. The entry point in the BRM capabilities depends on the specific output needed from the DM phase. The output from the discovery phase is a business decision architecture which consists of a Decision Requirements Diagram (DRD) and the underlying business logic [16]. The BRM capabilities which could be supported by the Discovery phase are: Elicitation, Design, and Specification. Depending on the specific level of detail needed from the Discovery output, the entry point is spread over these three phases.

\section{Elicitation}

A decision log is necessary to perform DM. Therefore, the elicitation capability will focus on finding and selecting a decision log. In this study, this is already done by selecting the Cardiovascular Risk Case (as shown in Figure 5) and the Dutch Teacher Grant case (as shown in Figure 6).

\section{Design}

The main purpose of the Design capability is to a create a business decision architecture from the relevant knowledge (a decision log) collected in the elicitation capability. This business rules architecture could be visualized through a modelling notation such as DMN.
The output from the discovery phase is, when adhering to DMN, a DRD. Therefore, this specific output of the Discovery phase could be specified under the Design capability.

For the Dutch Teacher Grant case, this results in the DRD as visualized in Figure 7.

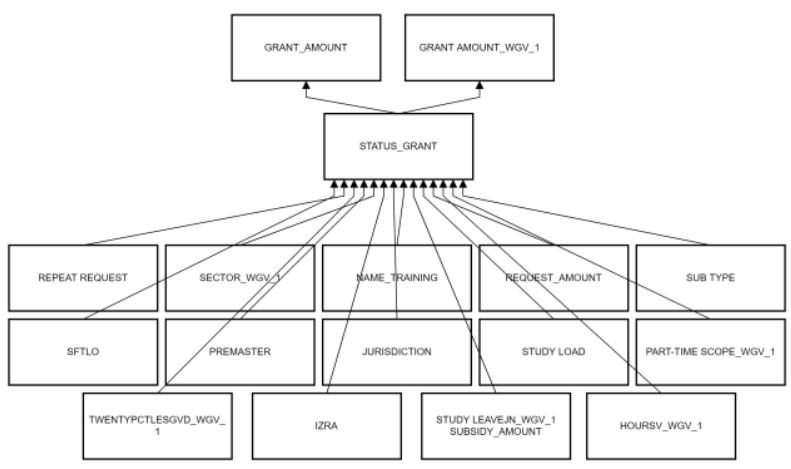

Figure 7. Dutch Teacher Grant Decision model

The DRD created out of the Dutch Teacher Grant case is visualized as such because of the many conditions that are used to determine whether the Teacher Grant can be handed out.

The Cardiovascular Risk case differs from the government case because consists of two decisions, as is visualized in Figure 8.

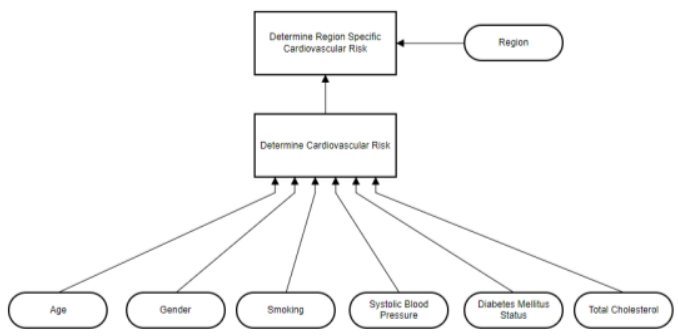

Figure 8. Cardiovascular Risk Case Decision model

The DRD visualizes the six conditions linked to the first decision and the dependency between the two decisions.

\section{Specification}

The main goal of the specification capability is to define business logic e.g., business rules or decision tables, the facttypes, and fact values for each business decision. Besides the DRD from the design capability, additional output is the underlying business logic. The Discovery phase utilizes the provided decision log to specify business rules and decision tables. For the Dutch Teacher Grant case the decision table is shown in Table 1. 
Table 1. Dutch Teacher Grant Decision Table

\begin{tabular}{|c|c|c|c|c|c|}
\hline & 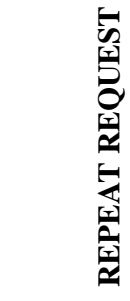 & 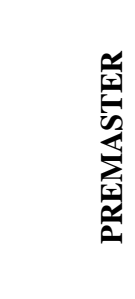 & 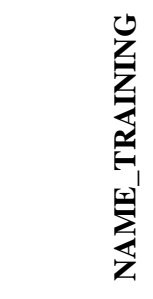 & $\vdots$ & 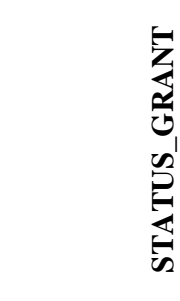 \\
\hline & Boolean & Boolean & String & $\ldots$ & String \\
\hline 1 & $\mathrm{Y}$ & $\mathrm{Y}$ & Master & $\ldots$ & APPROVED \\
\hline 2 & $\mathrm{~N}$ & $\mathrm{~N}$ & Bachelor & $\ldots$ & REJECTED \\
\hline
\end{tabular}

Where the Teacher Grant case consists of one decision table, the Cardiovascular Risk case consists of two decisions and therefore has two decision tables. The first decision table, shown in Table 2, shows the decision "Determine Cardiovascular Risk". The conclusion of this decision is used in the second decision table, shown in Table 3, where the cardiovascular risk is calculated for a specific subregion.

Table 2. Cardiovascular Risk Case Decision Table 1/2

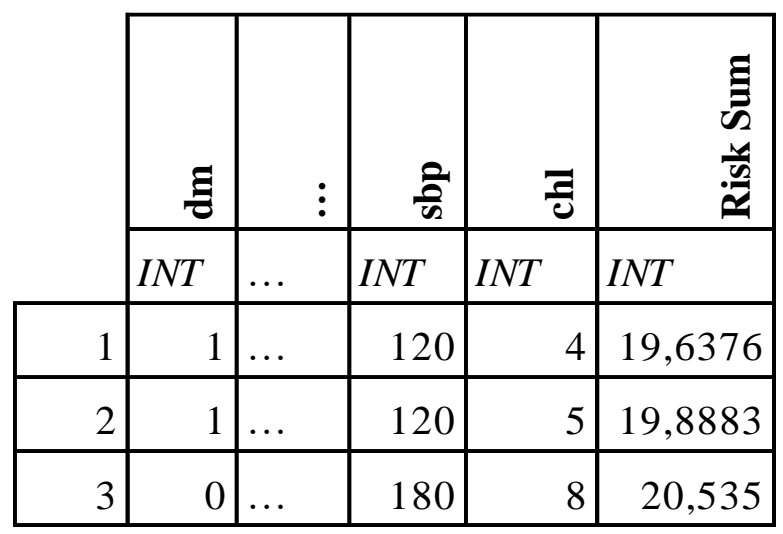

Table 3. Cardiovascular Risk Case Decision Table 2/2

\begin{tabular}{|c|c|c|}
\hline $\begin{array}{l}0 \\
.00 \\
00 \\
0 \\
0 \\
\text { D } \\
\end{array}$ & $\begin{array}{l}\Xi \\
\Xi \\
\tilde{n} \\
\vec{n} \\
\vec{n}\end{array}$ & 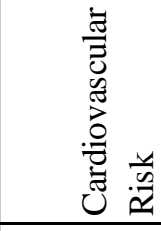 \\
\hline String & INT & FLOAT \\
\hline AFR_D & $<19,876$ & $<10 \%$ \\
\hline AFR_D & {$[20,3454 ; 20,5604]$} & $>=40 \%$ \\
\hline AMR_D & $<19,883$ & $<10 \%$ \\
\hline
\end{tabular}

\subsection{Conformance entry point}

The following entry point is that of Conformance checking. During this DM phase, a decision log is checked on conformance with a provided model. For instance, there may be a decision model indicating that a certain decision requires two checks before it goes through. Analysis of the decision log will show whether this business rule is followed or not.

This phase supports two BRM capabilities: The Verification and Validation capabilities. Verification aims on determining if artifacts adhere to predefined criteria and are logically consistent [6]. An integration can be made with the work of Corea et al. [11] to verify the decision tables. Validation aims towards determining whether the verified business decisions deliver their intended behavior [31]. The Conformance checking for the Dutch Teacher Grant case is shown in Figure 9. This shows that during the execution of a decision one out of eleven executions did not use two conditions: Sector_wgv_l and Twentypctlesgvd_wgv_l. The two unused conditions could be identified through the comparison of the decision $\log$, which are executed decisions, compared to the existing business decision architecture, which are decisions which should be used.

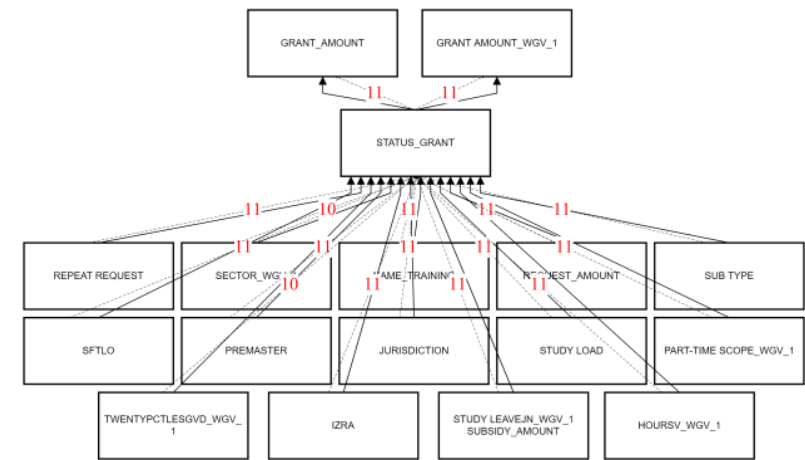

Figure 9. Dutch Teacher Grant Conformance checking

The Dutch Teacher Grant presented conformance on a DRD, but conformance checking can also be done on decision tables. Two decision tables with the business logic of the Cardiovascular Risk case are shown in Figure 10. In this example, anomalies are found and circled red between the theoretical model and the model created using decision discovery. Row three from the discovered model has a different conclusion comparing to the theoretical model while the input values are the same. 
Theoretical model

\begin{tabular}{|c|c|c|c|c|c|c|c|}
\hline & & 咅 & है & $\begin{array}{lll}8 \\
\infty \\
\sigma\end{array}$ & के & z & $\begin{array}{l}\bar{g} \\
\bar{n} \\
\underline{n} \\
\underline{a}\end{array}$ \\
\hline & & $I N T$ & $I N T$ & $I N T$ & $I N T$ & INT & $I N T$ \\
\hline & 1 & 0 & 1 & 40 & 120 & 4 & 19,6375578 \\
\hline & $\boldsymbol{I}$ & 0 & 1 & 40 & 120 & 5 & 19,8883042 \\
\hline 3 & 0 & 0 & 0 & 70 & 180 & 8 & 20,5349915 \\
\hline
\end{tabular}

Discovered model

\begin{tabular}{|c|c|c|c|c|c|c|c|}
\hline & $\Xi$ & $\vec{t}$ & है & 兽 & $\frac{0}{n}$ & 궁 & 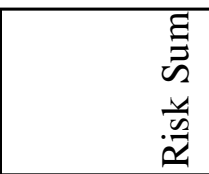 \\
\hline & $I N T$ & $I N T$ & $I N T$ & INT & $I N T$ & $\mid$\begin{tabular}{|l|}
$\mid I N T$ \\
\end{tabular} & $I N T$ \\
\hline & 1 & 0 & 1 & 40 & 120 & 4 & 19,6375578 \\
\hline 2 & 1 & 0 & 1 & 40 & 120 & 5 & 19,8883042 \\
\hline 3 & 0 & 0 & 0 & 70 & 180 & 8 & 20,2836771 \\
\hline
\end{tabular}

\section{Figure 10. Cardiovascular Risk Case} Conformance checking

\subsection{Improvement entry point}

Improvement is the last DM phase and focuses on providing possible improvements to the existing model and will eventually result into a new model. The BRM capabilities which could be supported are the Design and Specification capabilities. The Improvement phase for the Dutch Teacher Grant case is shown in Figure 11.

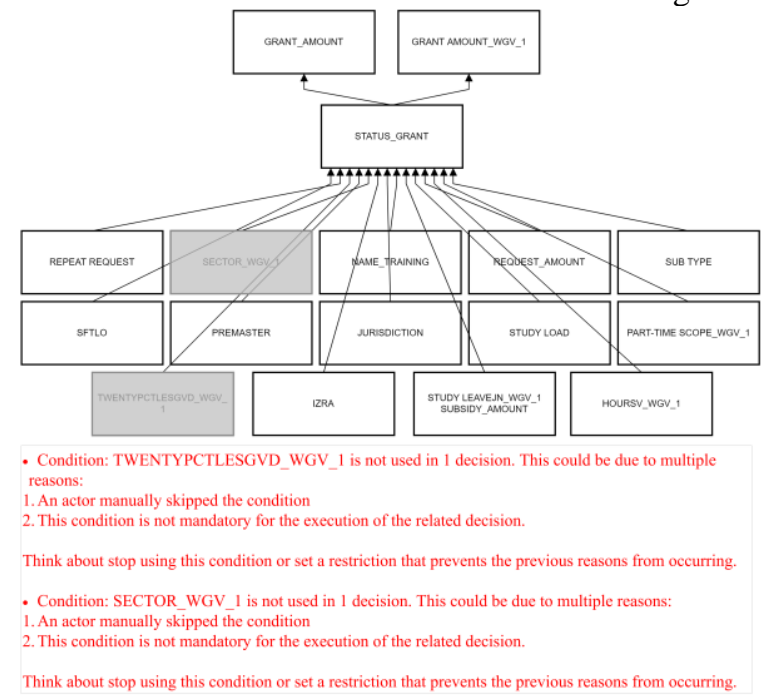

Figure 11. Dutch Teacher Grant Improvement

For the Dutch Teacher Grant case the improvement phase proposes possible solutions for a new model with the accompanying text:
Condition: TWENTYPCTLESGVD_WGV_1 is not used in 1 decision. This could be due to multiple reasons:

1. An actor manually skipped the condition

2. This condition is not mandatory for the execution of the related decision.

Think about stop using this condition or set a restriction that prevents the previous reasons from occurring.

Condition: SECTOR_WGV_1 is not used in 1 decision. This could be due to multiple reasons:

1. An actor manually skipped the condition

2. This condition is not mandatory for the execution of the related decision.

Think about stop using this condition or set a restriction that prevents the previous reasons from occurring.

In this case, removing two conditions because these are not utilized in earlier executed decisions.

For the Cardiovascular Risk case, the improvement phase shows a possible improvement for a new decision table. In this example, two rows with different conditions have exactly the same output. This is a partial reduction and can be identified during the verification of decision tables. However, the improvement phase also consists of proposing a new model instead of only identifying. The risk Sum of subregion EUR_A and AMR_D were modelled on separate rows, but the Cardiovascular Risk outcome is the same for both regions. Therefore, the two rows can be combined. The improvement phase proposes a new decision table where the two rows are combined, as depicted in red in Figure 12.

\begin{tabular}{|c|c|c|c|}
\hline & 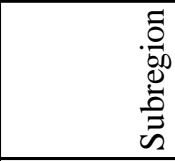 & 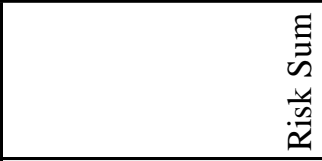 & 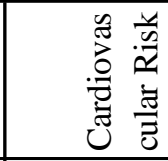 \\
\hline & String & INT & FLOAT \\
\hline 1 & AFR_D & $<19,876$ & $<10 \%$ \\
\hline 2 & AFR_D & {$[20,3454 ; 20,5604]$} & $>=40 \%$ \\
\hline 3 & AMR_D & $<19,883$ & $<10 \%$ \\
\hline 3 & EUR_A & $<19,883$ & $<10 \%$ \\
\hline
\end{tabular}




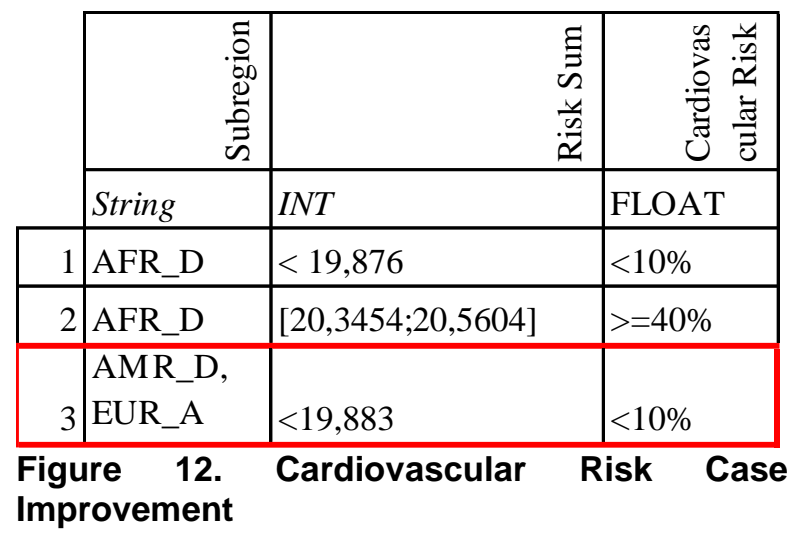

\section{Discussion and Future Research}

This study has several limitations. Firstly, the approach of this study is based on a conceptual simulation. Such an approach is appropriate given the fact that, to the knowledge of the authors, contributions in the body of knowledge aimed to integrate both research subdomains are not present. However, one could argue that further demonstration is required using computational simulation as well as adding empirical evidence of combining the presented DM phases and BRM capabilities in practice and the effects it has on the context of decision-making. Future research should therefore identify challenges with regards to integrating DM phases and BRM capabilities as well as to identify solutions for them. Based on such findings, researchers, practitioners, and organizations could collaborate on defining a reference process for the integration so that BRM processes are optimally utilizing the potential of DM phases.

Lastly, the technical integration of the DM phases in this study is a theoretical demonstration rather than a computational simulation. Currently, a large study is being conducted on the technical integration and possibilities of the discovery, conformance checking and improvement phases of decision mining [32]. More specific, the creation of algorithms for the discovery, conformance checking, and improvement of decisions. Research does exist on manually supporting specific BRM capabilities. For example, looking at conformance checking, where the study of [11] focuses on rule based checking. Future research should take this research into consideration when developing automatic conformance checking techniques as part of decision mining.

\section{Conclusion}

This study aimed on achieving the following research goal: To demonstrate how the phases of DM could be integrated with the BRM capabilities. To do so, we explored the phases with regards to DM and the capabilities with regards to BRM and proposed an integration by identifying possible entry points. These are demonstrated using two running examples using a conceptual simulation approach. Overall, from a conceptual level, the three DM phases 1) discovery, 2) improvement, and 3) conformance checking match with and enhance multiple BRM capabilities.

Entry point 1 features an integration between the discovery (DM) and elicitation, design and specification (BRM) capabilities, where a decision log is used to extract input for the identification of relevant sources and underlying input data, the business decision architecture and the underlying business logic.

Entry point 2 features an integration between the conformance checking (DM) phase and the verification and validation (BRM) capabilities, where a decision log is used to check for conformance against the business decision architecture as well as the underlying business logic featuring, e.g., business rules, facttypes and fact values.

Entry point 3: The DM improvement phase has a functional overlap with the design and specification BRM capabilities. Improvement supports the same capabilities as with entry point 1 , the difference lies with that entry point 1 results into a newly discovered model, and entry point 3 results into a improved model.

From a theoretical viewpoint, this study adds to the body of knowledge on how, from a higher level of abstraction, DM phases and BRM capabilities can be integrated. This contribution enables future research to be conducted into further examination of the integration proposed.

From a practical viewpoint, this study contributes towards practice in a sense that it triggers practitioners to explore how the upcoming developments of DM could be integrated into their BRM capabilities and processes. This could enable them to construct a better feedback-loop towards, e.g., regulatory institutions that create and publish sources, with the goal to improve the quality of law and regulations.

\section{References}

[1] van der Aalst, W.M.P., Process Mining, Springer Berlin Heidelberg, Berlin, Heidelberg, 2011.

[2] Bazhenova, E., S. Buelow, and M. Weske, "Discovering Decision Models from Event Logs", In Lecture Notes in Business Information Processing. Springer, 2016, 237251.

[3] Bazhenova, E., and M. Weske, "Deriving Decision Models from Process Models by Enhanced Decision Mining”, In Lecture Notes in Business Information Processing. 2016, 444-457.

[4] Berti, A., S.J. Van Zelst, and W. Van Der Aalst, "PM4Py web services: Easy development, integration and deployment of process mining features in any application 
stack", PM (PhD/Demos), (2019), 174-178.

[5] Blenko, M., M. Mankins, and P. Rogers, "The DecisionDriven Organization", Harvard Business Review(june), 2010, pp. 10.

[6] Boyer, J., and H. Mili, Agile Business Rule Development, Springer Berlin Heidelberg, Berlin, Heidelberg, 2011.

[7] Breaux, T.D., "Legal Requirements Acquisition for the Specification of Legally Compliant Information Systems", 2009, pp. 1-112.

[8] Collins, D., J. Lee, N. Bobrovitz, C. Koshiaris, A. Ward, and C. Heneghan, "whoishRisk - an R package to calculate WHO/ISH cardiovascular risk scores for all epidemiological subregions of the world", F1000Research 5, 2017, pp. 2522.

[9] Davis, J.P., K.M. Eisenhardt, and C.B. Bingham, "Developing Theory Through Simulation Methods", Academy of Management Review 32(2), 2007, pp. 480499.

[10] Graham, I., Business rules management and service oriented architecture a pattern language, John Wiley \& Sons, Hoboken, NJ, 2006.

[11] Hasić, F., C. Corea, J. Blatt, P. Delfmann, and E. Serral, "A Tool for the Verification of Decision Model and Notation (DMN) Models", In Lecture Notes in Business Information Processing. 2020, 536-542.

[12] Leewis, S., K. Smit, and M. Zoet, "Putting Decision Mining into Context: A Literature Study", In Lecture Notes in Information Systems and Organisation. 2020, 31-46.

[13] Leoni, M. de, and F. Mannhardt, "Decision Discovery in Business Processes", In Encyclopedia of Big Data Technologies. Springer International Publishing, Cham, 2018, 1-12.

[14] Morgan, T., Business Rules and Information Systems : Aligning IT with Business Goals, Addison-Wesley, Boston, MA, 2002.

[15] Nelson, M.L., R.L. Rariden, and R. Sen, "A Lifecycle Approach towards Business Rules Management", Proceedings of the 41st Annual Hawaii International Conference on System Sciences (HICSS 2008), IEEE (2008), 113-113.

[16] Object Management Group, Decision Model And Notation (DMN), Version 1.1, 2016.

[17] Rozinat, A., and W.M.P. van der Aalst, "Decision Mining in ProM", Business Process Management: 4th International Conference, BPM 2006, Vienna, Austria, September 5-7, 2006. Proceedings, Springer Berlin Heidelberg (2006), 420-425.

[18] Schlosser, S., E. Baghi, B. Otto, and H. Oesterle, "Toward a Functional Reference Model for Business Rules Management", 2014 47th Hawaii International Conference on System Sciences, IEEE (2014), 38373846.

[19] De Smedt, J., S.K.L.M. vanden Broucke, J. Obregon, et al., "Decision Mining in a Broader Context: An Overview of the Current Landscape and Future Directions", In Lecture Notes in Business Information Processing. Springer International Publishing, 2017, 197-207.

[20] De Smedt, J., F. Hasić, S.K.L.M. vanden Broucke, and J. Vanthienen, "Towards a Holistic Discovery of Decisions in Process-Aware Information Systems", International Conference on Business Process Management, 2017, pp. 183-199.

[21] De Smedt, J., F. Hasić, S.K.L.M. vanden Broucke, and J. Vanthienen, "Holistic discovery of decision models from process execution data", Knowledge-Based Systems 183, 2019, pp. 104866.

[22] Smirnov, A., M. Pashkin, T. Levashova, A. Kashevnik, and N. Shilov, "Context-Driven Decision Mining", In Encyclopedia of Data Warehousing and Mining. Information Science Reference, Hershey, NY, 2009, $320-327$.

[23] Smit, K., Organization and Governance of Business Rules Management Capabilities, Open University, 2018.

[24] Smit, K., and M. Zoet, "Management control system for business rules management", International Journal on Advances in Systems and Measurements (IARIA) 9(3), 2016, pp. 210-219.

[25] Smit, K., and M. Zoet, "An Organizational Capability and Resource-based Perspective on Business Rules Management", International Conference on Information Systems 2018, ICIS 2018, AIS Electronic Library (AISeL) (2018), 1-17.

[26] Smit, K., and M. Zoet, "Identifying Challenges in Business Rules Management Implementations Regarding the Governance Capability at Governmental Institutions", Proceedings of the 51st Hawaii International Conference on System Sciences, 2018.

[27] Smit, K., M. Zoet, and M. Berkhout, "Functional Requirements for Business Rules Management Systems", Twenty-third Americas Conference on Information Systems, 2017, pp. 1-10.

[28] Smit, K., M. Zoet, and M. Berkhout, "Verification capabilities for business rules management in the Dutch governmental context", 2017 International Conference on Research and Innovation in Information Systems (ICRIIS), IEEE (2017), 1-6.

[29] The Open Group, "TOGAF v9.1 standard", 2011. http://pubs.opengroup.org/architecture/togaf9-doc/arch/

[30] Zoet, M., Methods and Concepts for Business Rules Management, Hogeschool Utrecht, Utrecht, 2014.

[31] Zoet, M., and J. Versendaal, "Business Rules Management Solutions Problem Space: Situational Factors", Pacific Asia Conference on Information Systems 2013 (PACIS), (2013), 247.

[32] "NWO Research Grant", 2020. https://www.nwo.nl/projecten/023016003 